PROCEEDINGS OF THE AMERICAN MATHEMATICAL SOCIETY

Volume 135, Number 6, June 2007, Pages 1861-1863

S $0002-9939(07) 08697-2$

Article electronically published on January 5, 2007

\title{
ON THE ROLEWICZ THEOREM FOR EVOLUTION OPERATORS
}

\author{
K. V. STOROZHUK
}

(Communicated by Joseph A. Ball)

\begin{abstract}
We give a short proof of a generalization of the Rolewicz theorem based on the uniform boundedness principle.
\end{abstract}

We say that a function $f:(0, \infty) \rightarrow(0, \infty)$ is proper if $f$ is nondecreasing.

Theorem 1. Suppose that $X$ is Banach space, and a $C_{0}$-semigroup $\left\{T_{t}: X \rightarrow X\right\}$ is not exponentially bounded, i.e. $\left\|T_{t}\right\|$ does not decrease exponentially as $t \rightarrow \infty$. For each proper function $f$, there exists $x \in X$ such that $\int_{0}^{\infty} f\left(\left|T_{t}(x)\right|\right) d t=\infty$.

R. Datko proved this fact in 2] for $f(z)=z^{2}$ and $X$ a Hilbert space. (There he established an analog to the Liapounov theorem.) A. Pazy generalized it in [4 for $f(z)=z^{p}, p \in[1, \infty)$. For continuous strictly monotone proper functions, this fact was obtained by Littman 3 .

Moreover, Rolewicz [7] considered evolution operators (satisfying $U(t, s) U(s, r)=$ $U(t, r), U(t, t)=\operatorname{Id}$ and $U(t, s)(x)$ is continuous with respect to $t$ for each $x \in X)$.

Theorem 2 (Rolewicz). Suppose that $U(t, s): X \rightarrow X, t \geq s \geq 0$, are evolution operators on a Banach space $X$, which are uniformly bounded but not uniformly exponentially bounded (i.e. $\sup _{s>0}\|U(s+p, s)\|$ does not decrease exponentially in $p)$. Assume that $N(\alpha, u): \mathbb{R}_{+} \times \mathbb{R}_{+} \rightarrow \mathbb{R}_{+}$is continuous and the function $f_{\alpha}(u):=N(\alpha, u)$ is proper for every $\alpha$. Then there is $x \in X$ such that for all $\alpha$

$$
\sup _{s<\infty} \int_{0}^{\infty} f_{\alpha}(|U(s+p, s)(x)|) d p=\infty .
$$

Rolewicz-type theorems were proved, for example, in [1, 8].

We establish a Rolewicz-like result in the next theorem.

Theorem 3. Suppose that $U(t, s)$ are evolution operators on a Banach space $X$, which are uniformly bounded but not uniformly exponentially bounded. If $f_{\alpha}(u)=$ $N(\alpha, u), \alpha \in \mathbb{R}^{m}$, is continuous in $\alpha$ and proper on $u$, then there is $x \in X$ such that the condition (1) is fulfilled for each $f_{\alpha}, \alpha \in \mathbb{R}^{m}$.

Note that in [8] the assumption of continuity on $u$ was also removed.

Received by the editors December 16, 2005 and, in revised form, February 14, 2006. 2000 Mathematics Subject Classification. Primary 47D06; Secondary 34G10.

Key words and phrases. $C_{0}$-semigroup, evolution operator, uniform exponential stability.

The author was supported by the Program of Russian Federation, Grant NSh-8526.2006.1.

(C)2007 American Mathematical Society Reverts to public domain 28 years from publication 
Lemma 4. Let $T_{n}: X \rightarrow X$ be linear operators on a Banach space with $\left\|T_{n}\right\| \not \rightarrow 0$. For each positive sequence $a_{n} \rightarrow 0$, there exists $x$ such that $\overline{\lim }\left\{\frac{\left|T_{n}(x)\right|}{a_{n}}\right\}=\infty$. If $f$ is a proper function and $b_{n} \rightarrow \infty$, then there is $x$ such that $\overline{\lim } b_{n} f\left(\left|T_{n}(x)\right|\right)=\infty$.

Proof. Apply the Banach-Steinhaus Theorem for the unbounded set of operators $\left\{\frac{T_{n}}{a_{n}}\right\}$. To prove the second part of the lemma, note that if $f$ is a proper function, then there is a sequence $a_{n} \rightarrow 0$ such that $f\left(a_{n}\right) \geq\left(b_{n}\right)^{-\frac{1}{2}}$ for large $n$. Now if $\left|T_{n_{k}}(x)\right| \geq a_{n_{k}}$, then $b_{n_{k}} \cdot f\left(\left|T_{n_{k}}(x)\right|\right) \geq b_{n_{k}} \cdot f\left(a_{n_{k}}\right) \geq \sqrt{b}_{n_{k}} \rightarrow \infty$.

Lemma 5. If $f_{\alpha}(u)=N(\alpha, u), \alpha \in \mathbb{R}^{m}$, is continuous in $\alpha$ and proper on $u$, then there is a proper $f$ such that $f(u) \leq f_{\alpha}(u)$ as $u \rightarrow 0$ for each $\alpha \in \mathbb{R}^{m}$.

Proof. Cover $\mathbb{R}^{m}$ by compacts $K_{1} \subset K_{2} \subset \ldots$ and put $f_{n}=\inf \left\{f_{\alpha} \mid \alpha \in K_{n}\right\}$. Then $f_{n}$ are proper functions and $f_{1} \geq f_{2} \geq \ldots$ Now for $u \in\left(\frac{1}{n+1}, \frac{1}{n}\right]$ put $f(u)=f_{n}(u)$.

Proof of Theorem 3. Let $f$ be as in Lemma 5. If condition (1) is fulfilled for $f$, then the same condition is fulfilled for each $f_{\alpha}, \alpha \in \mathbb{R}^{m}$.

Note that $\sup _{s}\{\|U(p+s, s)\|\}=\lambda_{p} \geq 1$ for every $p \geq 0$. Otherwise the family $\{U\}$ would be exponentially bounded, since

$$
\|U(p n+s, s)\| \leq\|U(p n+s, p(n-1)+s) \circ \cdots \circ U(p+s, s)\| \leq \lambda_{p}^{n}
$$

and $\|U(t+s, s)\| \underset{t \rightarrow \infty}{=} O\left(e^{\frac{\ln \lambda_{p}}{p} t}\right)$. In particular, there exists a sequence $s_{n}$ such that $\left\|U\left(n+s_{n}, s_{n}\right)\right\| \not \rightarrow 0$ as $n \rightarrow \infty$.

Put $c=\sup \{\|U\|\}$. From composition law we see that if $n \geq p$, then for each $s\|U(n+s, s)\| \leq c\|U(p+s, s)\|$. Then $|U(p+s, s)(x)| \geq\left|U(n+s, s)\left(\frac{x}{c}\right)\right|$, and we have

$$
\int_{0}^{\infty} f\left(\left|U\left(p+s_{n}, s_{n}\right)(x)\right|\right) d p \geq \int_{0}^{n} f\left(\left|U\left(p+s_{n}, s_{n}\right)(x)\right|\right) d p \geq n \cdot f\left(\left|U\left(n+s_{n}, s_{n}\right)\left(\frac{x}{c}\right)\right|\right) .
$$

By Lemma 4, the right-hand side of the inequality is unbounded on some $x$.

Remark 1. Lemma 4 uses only the uniform boundedness principle, therefore Theorem 5 is valid for normed barreled spaces and for Frechet spaces (if we replace $|x|$ by $\rho(0, x)$ for $x \in X)$. Also we can replace $\mathbb{R}^{m}$ by any $\sigma$-compact $Y$.

Remark 2. Van Neerven [6] generalized the Datko-Pazy theorem in another direction, by replacing $\int$ with a more general functional. He made use of an assertion in 5] which is much stronger than our Lemma 4 (van Neerven's result implies that the first assertion of Lemma 4 still holds even if we replace "lim" by "씨").

The author wishes to thank E. Emelyanov and J. M. A. M. van Neerven for their communications.

\section{REFERENCES}

[1] C. Buse, S. Dragomir, New characterizations of asymptotic stability for evolution families on Banach spaces, Electron. J. Differential Equations 2004, 38, 9 pp. (electronic). MR2047394 (2005b:34112)

[2] R. Datko, Extending a theorem of A. M. Liapounov to Hilbert space, J. Math. Anal. Appl. 32 (1970), 610 - 616. MR 0268717 (42:3614)

[3] W. Littman, A generalization of a theorem of Datko and Pazy, in Lecture Notes in Control and Inform. Sci., v. 130, Springer-Verlag, Berlin, 1989, pp. 318-323. MR1029070 (91a:47052) 
[4] A. Pazy, On the applicability of Lyapunov's theorem in Hilbert Space, SIAM J. Math. Anal., v.3 2 (1972), 291-294. MR0317105 (47:5653)

[5] J. M. A. M. van Neerven, On the orbits of an operator with spectral radius one, Czechoslovak Math. J. 45(120) (1995), 3, 495-502. MR.1344516 (96f:47009)

[6] J. M. A. M. van Neerven, M. Lower semicontinuity and the theorem of Datko and Pazy, Integral Equations Operator Theory 42 (2002), 4, 482-492. MR.1885446 (2003i:47044)

[7] S. Rolewicz, On uniform N-equistability, J. Math. Anal. Appl. 115 (1986), 434-441. MR0836237 (87h:47100)

[8] Q. Zheng, Exponential stability and perturbation problems for linear evolution systems in Banach spaces (Chinese, English Review), J. Sichuan Univ. 25 (1988), 4, 401-411. MR0995048 (90e:34110)

Sobolev Institute of Mathematics, Acad. Koptyug Pr. 4, 630090 Novosibirsk, Russia 